\title{
Accuracy of Visual Assessment for Quantification of Myocardial Edema Extension in Takotsubo Syndrome.
}

\author{
Alberto Cecconi ${ }^{1}$, Jorge Salamanca ${ }^{1}$, Teresa Alvarado ${ }^{1}$, Paula Antuna ${ }^{1}$, Susana Hernandez \\ Muñiz ${ }^{1}$, Maria Jose Olivera ${ }^{1}$, Paloma Caballero ${ }^{1}$, Luis Jesus Jimenez-Borreguero ${ }^{1}$, and \\ Fernando Alfonso ${ }^{1}$ \\ ${ }^{1}$ Hospital Universitario La Princesa
}

April 28, 2020

\begin{abstract}
The aim of this study was to define the correlation between visual qualitative method and semi-quantitative method for the study of the extension of myocardial edema by CMR in patients with Takotsubo syndrome (TTS). We analyzed 22 consecutive patients with a diagnosis of typical apical TTS. A total of 374 myocardial segments were analyzed. A high correlation between visual method and the semi-quantitative method of the T2 SI ratio (Pearson's $\mathrm{r}=0.82, \mathrm{p}<0.001$ ) was found. In the clinical setting the visual method provides a valid and reliable quantification of myocardial edema extension in patients with TTS.
\end{abstract}

Abstract:

The aim of this study was to define the correlation between visual qualitative method and semi-quantitative method for the study of the extension of myocardial edema by CMR in patients with Takotsubo syndrome (TTS).

We analyzed 22 consecutive patients with a diagnosis of typical apical TTS. A total of 374 myocardial segments were analyzed. A high correlation between visual method and the semi-quantitative method of the T2 SI ratio (Pearson's $\mathrm{r}=0.82, \mathrm{p}<0.001$ ) was found.

In the clinical setting the visual method provides a valid and reliable quantification of myocardial edema extension in patients with TTS.

Background . Myocardial edema is characteristic of the acute phase of Takotsubo syndrome (TTS) and can be easily detected by cardiac magnetic resonance (CMR) [1]. In conventional edema sequences, the presence and extension of edema can be assessed qualitatively by visual analysis of the signal intensity (SI) or semi-quantitatively by analyzing the T2 SI ratio between myocardial and skeletal muscle [2]. In the clinical practice the qualitative method is widely accepted in the scenario of acute myocardial infarction, early stages of myocarditis and sarcoidosis [3]. However, whether a quantitative method should be preferred in patients with TTS remains unknown. The aim of this study was to define the correlation between visual qualitative method and semi-quantitative method for the study of the extension of myocardial edema in patients with TTS.

Methods. We included all consecutive patients admitted between January 2013 and March 2016 with a diagnosis of typical apical TSS (according to the criteria of the Mayo Clinic), following a prospective comprehensive and systematic protocol including a CMR study in the acute phase [4]. CMR protocol included a specific T2-weighted sequence (double inversion recovery spin echo with fat suppression) on short- and long-axis (2-,3-, and 4-chamber) views in a 1.5 Tesla MRI Scanner (Signa HDXT; General Electric Healthcare(R, Milwaukee, WI, USA), using a 12-channel phased array surface coil and ECG synchronization. 
All patients provided informed consent to participate in the study. The extent of the edema was assessed by accredited experts and expressed as number of myocardial segments involved using the 17-segments cardiac segmentation model. The quantification of the edema was performed both by visual SI and by the semiquantitative T2 SI ratio. For the T2 SI ratio, a ROI was drawn in every myocardial segment and then compared with a ROI localized in the skeletal muscle closest to the heart and to the center of the reception field of the coil. A value $>1.9$ in the T2 SI ratio was considered positive for the presence of edema in the myocardial segment. Patients negative for edema by T2 SI ratio were excluded. All the analyses were blind and independent.

Results . 22 patients were included in the analysis. A total of 374 myocardial segments were analyzed. The median of myocardial segments with edema was 5 (1-11) using the qualitative method and 6 (0-12) using the semi-quantitative method. The results showed a positive correlation between the extent of myocardial edema measured by the visual method and the semi-quantitative method of the T2 SI ratio (Pearson's $\mathrm{r}=$ $0.82, \mathrm{p}<0.001$ ) (Figure).

Discussion . Postprocessing guidelines support visual analysis for the assessment of myocardial edema in myocardial infarction, however they do not state whether this is also appropriate for TTS [3]. T2 SI ratio and T2 mapping are more accurate than visual assessment [5], but their use is limited in clinical practice because they are time consuming and the analyses are not widely accessible. Considering that visual edema assessment by CMR is the most common used method in the differential diagnosis of myocardial infarction with nonobstructive coronary arteries (MINOCA) and that TTS may represent up 20\% of MINOCA patients [6], we considered mandatory to test the accuracy of visual assessment in TTS with a more rigorous methods as T2 SI ratio.

Conclusions. The visual qualitative method presents a high correlation with T2 SI ratio in the study of myocardial edema by CMR in patients with TTS. Therefore, in the clinical setting the visual method provides a valid and reliable quantification of myocardial edema extension in patients with TTS.

\section{Author contributions:}

Concept/design: AC, JS, LJJB, FA; Data analysis/interpretation: AC, JS, SHM, MJO, PC; Drafting article: AC, JS, LJJB, FA, Critical revision of article: AC, TA, PA, SHM, MJO, PC, JS, LJJB, FA; Approval of article: AC, TA, PA, SHM, MJO, PC, JS, LJJB, FA; Statistics: AC, JS; Data collection: AC, JS, TA, PA.

\section{References :}

1. Salamanca, J., et al.,Temporal Resolution Pattern of Myocardial Edema in Patients With Takotsubo Syndrome. J Card Fail, 2018. 24 (5): p. 345-346.

2. Eitel, I. and M.G. Friedrich,T2-weighted cardiovascular magnetic resonance in acute cardiac disease. J Cardiovasc Magn Reson, 2011. 13 : p. 13.

3. Schulz-Menger, J., et al.,Standardized image interpretation and post processing in cardiovascular magnetic resonance: Society for Cardiovascular Magnetic Resonance (SCMR) board of trustees task force on standardized post processing. J Cardiovasc Magn Reson, 2013. 15 : p. 35.

4. Cecconi, A., et al.,Predictors of oedema in Tako-Tsubo cardiomyopathy: new insights into the diabetes paradox. J Cardiovasc Med (Hagerstown), 2019.20 (6): p. 406-408.

5. Messroghli, D.R., et al., Clinical recommendations for cardiovascular magnetic resonance mapping of T1, T2, T2* and extracellular volume: A consensus statement by the Society for Cardiovascular Magnetic Resonance (SCMR) endorsed by the European Association for Cardiovascular Imaging (EACVI). J Cardiovasc Magn Reson, 2017. 19 (1): p. 75.

6. Tamis-Holland, J.E. and H. Jneid, Myocardial Infarction With Nonobstructive Coronary Arteries (MINOCA): It's Time to Face Reality! J Am Heart Assoc, 2018.7 (13).

Figure legends: 
Figure. Correlation between the extent of myocardial edema measured by the visual method and the semiquantitative method.

On the left, the regression line is showed. On the right, mid-ventricular short-axis view, assessment by both methods is presented.
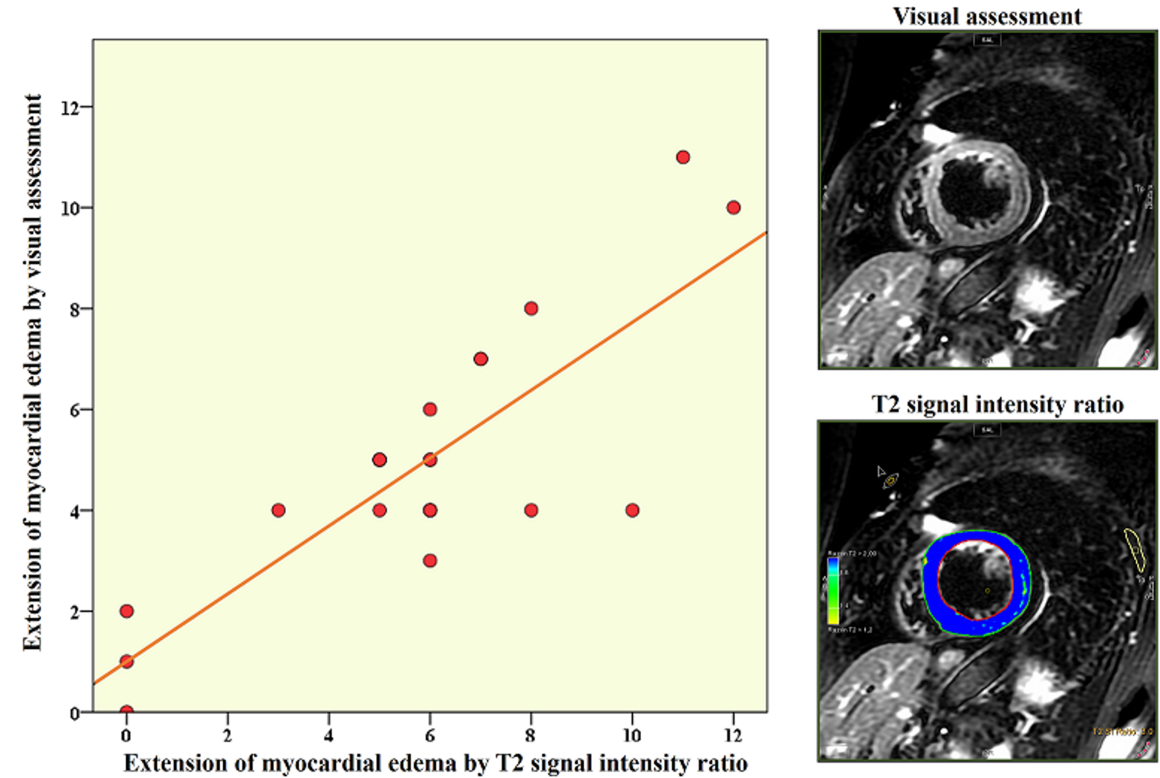